\title{
Adaptive nonlinear contour coupling control for a machine tool system
}

\section{Jinho Lee, Warren E. Dixon \& John C. Ziegert}

The International Journal of Advanced Manufacturing Technology

ISSN 0268-3768

Volume 61

Combined 9-12

Int J Adv Manuf Technol (2012)

61:1057-1065

DOI 10.1007/s00170-011-3760-1

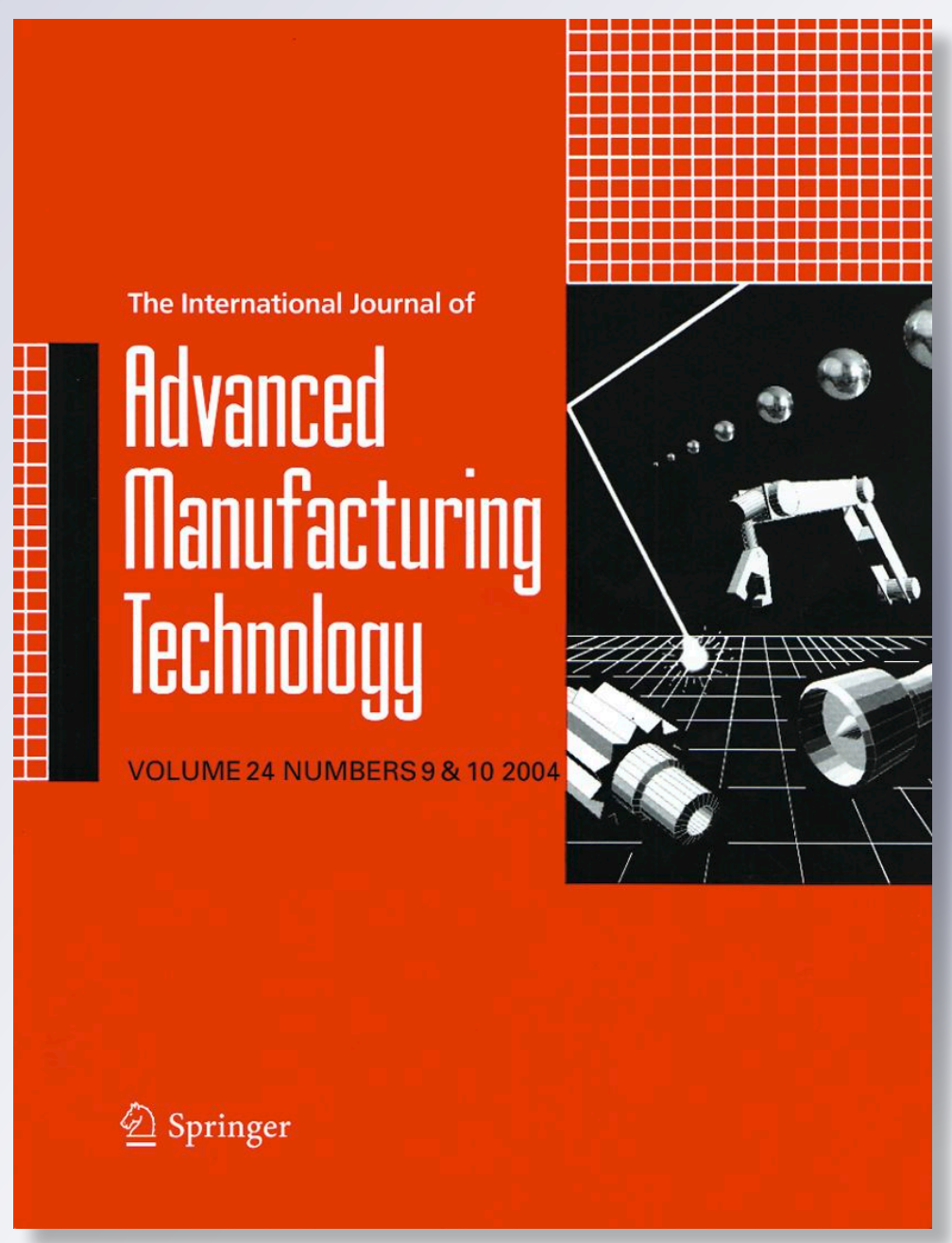

\section{望 Springer}


Your article is protected by copyright and all rights are held exclusively by Springer-Verlag London Limited. This e-offprint is for personal use only and shall not be self-archived in electronic repositories. If you wish to selfarchive your work, please use the accepted author's version for posting to your own website or your institution's repository. You may further deposit the accepted author's version on a funder's repository at a funder's request, provided it is not made publicly available until 12 months after publication. 


\title{
Adaptive nonlinear contour coupling control for a machine tool system
}

\author{
Jinho Lee · Warren E. Dixon · John C. Ziegert
}

Received: 17 March 2011 / Accepted: 7 November 2011 / Published online: 4 December 2011

(C) Springer-Verlag London Limited 2011

\begin{abstract}
The quality of products from a machine tool system is largely determined by the tolerances maintained, which is a function of how well the desired contour is tracked. To mitigate contour errors in a three-axis machine tool feed drive system, the control development in this paper is based on an error system that is transformed into tangential, normal, and binormal components to the desired contour (i.e., a crosscoupling controller (CCC)). Unlike previous CCCs, the controller developed in this paper does not assume exact knowledge of the inertia and friction matrices. Specifically, an adaptive estimate is developed to compensate for uncertain friction and inertial parameters. Lyapunov-based methods are used to craft the adaptive estimate and to prove global asymptotic contour tracking. Experimental results of the proposed controller on the $x-y$-axes of the high speed milling machine showed improvement of the contouring accuracy compared to proportional-derivative controller and a benchmark CCC.
\end{abstract}

Keywords Feed drive system • High speed machining • Contour error - Cross-coupling controller .

Adaptive estimate $\cdot$ Lyapunov control

\section{J. Lee $(\varangle)$}

Defense Agency for Technology and Quality, Seoul,

Republic of Korea

e-mail: jinholee74@gmail.com

W. E. Dixon

Mechanical and Aerospace Engineering, University of Florida, Gainesville, FL, USA

J. C. Ziegert

Mechanical Engineering, Clemson University, Clemson, $\mathrm{SC}, \mathrm{USA}$

\section{Introduction}

The contour error in computer numerical control (CNC) machine tool systems is defined as the error component orthogonal to a desired tool path. The contour error is a key factor used to quantify the quality of the machined product. To reduce the contouring error, historical controllers have focused on the improvement of the individual axis performance only, resulting in a simple single input single output (SISO) system. The individual axis is typically controlled through a proportional integral derivative strategy; however, some inverse dynamics-based feedforward controllers have also been proposed to eliminate the axial tracking error. Example feedforward controllers include the zero phase error tracking controller (ZPETC) [1] and the inverse compensation filter [2]. Also, recently, robust controllers focus on rejecting uncertainties in the drive parameters, maximizing the bandwidth within the physical limitations of the system, and compensating for external disturbances. The main drawback of methods that consider the performance of each axis separately during contouring is that reducing the individual axis errors does not necessarily reduce the contour error during nonlinear motions.

To address this problem, the cross-coupling controller (CCC) concept was introduced by Koren in [3] and [4]. The CCC objective is to mitigate the contour error rather than reducing the individual axis errors. The cross-coupling concept requires the construction of a contour error model that is used in the control development. Specifically, CCC are developed by transforming the inertial coordinate system to a coordinate system that moves along a desired contour. Through this transformation, the error between the current 
coordinates and the desired coordinates is decoupled into normal, tangential, and binormal components. This decoupling allows the normal error (i.e., the contour error) to be regulated independently from the tracking error (i.e., the tangential error).

In [5] and [6], Yeh and Hsu combined the CCC strategy with the ZPETC method using a contouring error transfer function. The resulting linear SISO error system was proven to yield bounded-input boundedoutput stability. Yeh and Hsu also proposed a modified variable-gain CCC in [7] that directly applies to arbitrary contours. Specifically, a linear contour approximation is used in lieu of the actual contour error as a means to reduce the computational complexity.

Srinivasan and Kulkarni developed and experimentally tested an optimal controller based on a linear feed drive model for a multi-axis feed drive system [8]. One conclusion stated in [8] is that improved performance may be obtained by designing the controller using a higher order linear model. Moreover, [8] stated that the unmodeled nonlinear dynamics seemed to play a significant role in the experimental demonstration. A $\mathrm{CCC}$ for a biaxial feed drive system was also developed and experimentally tested in [9]. However, the CCC in [9] was still designed based on a linear model of the machine. In [10], Chen et al. used a polar coordinate representation of the contour error so that a linear relationship between the contour error and the radial position can be developed. Through the use of the polar coordinate representation, the control objective in [10] is formulated as a stabilization problem for which a feedback linearizing controller was developed.

Difficulties in obtaining an accurate nonlinear model of cross-coupled multi-axis machine tools motivated Tarng et al. to employ a fuzzy logic feedforward control component in [11] and [12]. Degraded contour tracking at high-speed feedrates due to the nonlinear cross-coupled dynamics motivated Chuang and Liu to develop an adaptive estimate in [13]. The adaptive estimate was designed as a linear perturbed error model described by a deterministic autoregressive moving average. An adaptive CCC feedforward scheme was also proposed in [14]; however, as stated in [15], the approach is limited to a linear coordination objective (i.e., the desired contour is a linear relationship between the axes). Motivated by the limitations in [14], Chiu and Tomizuka formulated the contour tracking problem in a task coordinated frame in [15]. Specifically, the result in [15] encodes the desired contour as a combination of a feedrate, velocity direction, and instantaneous curvature, eliminating the need for an analytical contour representation. The controller was developed in [15] for a linear time-varying error system under the assump- tion of exact model knowledge. Also under the assumption of exact model knowledge of the dynamics except for an additive bounded disturbance, [16] developed an adaptive robust contour tracking controller where the problem was formulated as a regulation objective. More recently, [17] developed another adaptive robust controller using a discontinuous adaptive estimate for a linear motor driven $X-Y$ table with uncertainty in the nonlinear dynamics.

The CCC development in this paper is inspired by the task coordinated problem formulation introduced in the recent efforts in [15]. Lyapunov-based controllers are developed for a three-axis CNC machine tool feed drive system, although the results can be extended to $n$-dimensional systems. The contribution of the results over previous CCCs are that uncertainty in the resulting transformed machine tool dynamics is considered. Specifically, standard adaptive nonlinear control methods are applied to the transformed cross-coupled machine dynamics to compensate for uncertain inertial and friction parameters. A Lyapunov-based analysis is used to prove global asymptotic contour tracking for an arbitrary contour. The developed controller is applied to a feed drive system on a high speed milling machine (HSM2) in the University of Florida Machine Tool Research Center. Experiments are performed to illustrate the contouring performance of the proposed controller compared to a proportional and derivative (PD) controller and the benchmark CCC in [4].

\section{Three-axis CNC machine tool dynamics}

The dynamic model for a three-axis CNC machine tool is assumed to have the following form:

$M \ddot{x}+B \dot{x}=T$

where $x(t), \dot{x}(t), \ddot{x}(t) \in \mathbb{R}^{3}$ denote the position, velocity, and acceleration of the machine tool expressed in an inertial coordinate system, respectively; $M \in \mathbb{R}^{3 \times 3}$ denotes the unknown diagonal inertia matrix; $B \in \mathbb{R}^{3 \times 3}$ denotes the unknown diagonal friction matrix; and $T(t) \in \mathbb{R}^{3}$ represents the input control force. To facilitate the subsequent CCC development, the machine tool coordinates can be transformed from the inertial coordinate system to a time-varying coordinate frame as follows:

$x_{\mathrm{f}}=F^{T} x$

where $x_{\mathrm{f}}(t), \dot{x}_{\mathrm{f}}(t), \ddot{x}_{\mathrm{f}}(t) \in \mathbb{R}^{3}$ denote the position, velocity, and acceleration of the machine tool expressed in the time-varying coordinate system, respectively. The 
subsequent development is based on the assumption that $x_{\mathrm{f}}(t)$ and $\dot{x}_{\mathrm{f}}(t)$ are measurable. In Eq. 2, the known transformation matrix $F(t) \in \mathbb{R}^{3 \times 3}$ is composed of a known unit tangent vector, denoted by $t(t) \in \mathbb{R}$; a known unit normal vector, denoted by $n(t) \in \mathbb{R}$; and a known binormal vector, denoted by $b(t) \in \mathbb{R}$, defined as $b(t)=t(t) \times n(t)$. The vectors $t(t), n(t)$, and $b(t)$ are defined based on the tangent and normal components of a desired contour, denoted by $x_{\mathrm{d}}(t)$. That is, given a desired contour $x_{\mathrm{d}}(t)$, the tangential, normal, and binormal components of $x_{\mathrm{d}}(t)$ can be used to change the coordinates of the machine tool from the inertial reference frame to a time varying reference frame as follows:

$x_{\mathrm{fd}}=F^{T} x_{\mathrm{d}}$

where $x_{\mathrm{fd}}(t), \dot{x}_{\mathrm{fd}}(t), \ddot{x}_{\mathrm{fd}}(t) \in \mathbb{R}^{3}$ denote the desired position, velocity, and acceleration of the machine tool expressed in the time-varying coordinate system, respectively. See Fig. 1.

Based on Eqs. 2 and 3, the dynamic model given in Eq. 1 can be transformed as follows:

$\bar{M} \ddot{x}_{\mathrm{f}}+\bar{V}_{m} \dot{x}_{\mathrm{f}}+\bar{B} \dot{x}_{\mathrm{f}}+N x_{\mathrm{f}}=\bar{T}$.

In Eq. $4, \bar{M}(t), \bar{V}_{m}(t), \bar{B}(t), \quad N(t)$, and $\bar{T}(t)$ are defined as

$$
\begin{aligned}
\bar{M} & =F^{T} M F \\
\bar{V}_{m} & =-2 F^{T}\left(M F \dot{F}^{T}\right) F \\
\bar{B} & =F^{T} B F
\end{aligned}
$$

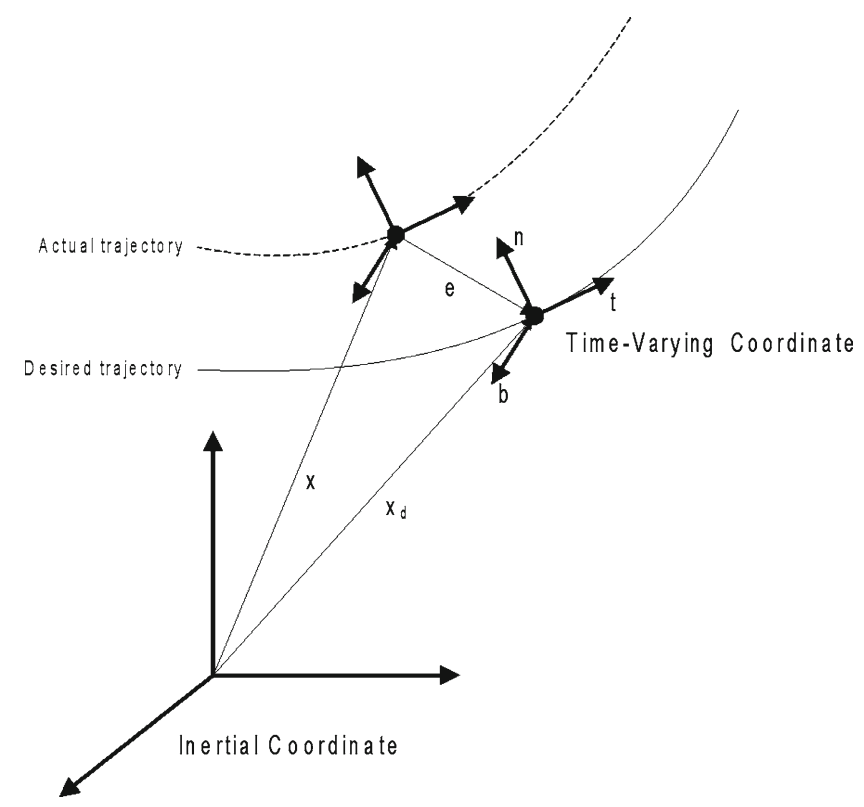

Fig. 1 Time-varying coordinate frame

$$
\begin{aligned}
N= & 2 F^{T}\left(M F \dot{F}^{T}\right) F\left(\dot{F}^{T} F\right) \\
& -F^{T} B F\left(\dot{F}^{T} F\right)-F^{T} M F \ddot{F}^{T} F
\end{aligned}
$$

$\bar{T}=F^{T} T$.

The dynamic model in Eq. 4 exhibits the following properties that are utilized in the subsequent development.

Property 1 The inertia matrix $\bar{M}(t)$ is symmetric, positive definite, and satisfies the following inequalities [18]

$m_{1}\|\xi\|^{2} \leq \xi^{T} \bar{M} \xi \leq m_{2}\|\xi\|^{2} \quad \forall \xi(t) \in \mathbb{R}^{3}$

where $m_{1}, m_{2} \in \mathbb{R}$ are known positive constants and $\|\cdot\|$ denotes the standard Euclidean norm.

Property 2 The matrices $\bar{M}(t)$ and $\bar{V}_{m}(t)$ are skewsymmetric in the sense that [18]

$\xi^{T}\left(\dot{\bar{M}}(t)-\bar{V}_{m}(t)\right) \xi=0 \quad \forall \xi(t) \in \mathbb{R}^{3}$.

Property 3 The dynamic model given in Eq. 4 can be linearly parameterized as follows [18]:

$Y \theta=\bar{M} \ddot{x}_{\mathrm{f}}+\bar{V}_{m} \dot{x}_{\mathrm{f}}+\bar{B} \dot{x}_{\mathrm{f}}+N x_{\mathrm{f}}$

where $\theta \in \mathbb{R}^{6}$ contains the unknown constant system parameters and $Y\left(x_{\mathrm{f}}, \dot{x}_{\mathrm{f}}, \ddot{x}_{\mathrm{f}}\right) \in \mathbb{R}^{3 \times 6}$ denotes a regression matrix. The regression matrix formulation of Eq. 12 can also be written in terms of the desired contour in the following manner:

$Y_{\mathrm{d}} \theta=\bar{M} \ddot{x}_{\mathrm{fd}}+\bar{V}_{m} \dot{x}_{\mathrm{fd}}+\bar{B} \dot{x}_{\mathrm{fd}}+N x_{\mathrm{fd}}$

where the desired regression matrix is defined by $Y_{\mathrm{d}}\left(x_{\mathrm{fd}}, \dot{x}_{\mathrm{fd}}, \ddot{x}_{\mathrm{fd}}\right) \in \mathbb{R}^{3 \times 6}$.

Property 4 The time derivative of the transformation matrix $F(t)$ can be expressed as follows [15]:

$\dot{F}^{T}=v R F^{T}$

where $v(t)=\left\|\dot{x}_{\mathrm{fd}}(t)\right\| \in \mathbb{R}$ denotes the desired feedrate and $R(t) \in \mathbb{R}^{3 \times 3}$ is defined as

$R=\left[\begin{array}{ccc}0 & \kappa & 0 \\ -\kappa & 0 & \tau \\ 0 & -\tau & 0\end{array}\right]$.

In Eq. $15, \kappa(t) \in \mathbb{R}$ is a single-valued continuous function that denotes the desired curvature of the contour, and $\tau(t) \in \mathbb{R}$ is a single-valued continuous function that denotes the desired torsion. The time derivative of Eq. 14 can be determined as

$\ddot{F}^{T}=\dot{v} R F^{T}+v \dot{R} F^{T}+v^{2} R R F^{T}$. 
Based on the assumption that the desired contour is selected such that $x_{\mathrm{fd}}(t), \dot{x}_{\mathrm{fd}}(t), \ddot{x}_{\mathrm{fd}}(t), \kappa(t), \tau(t), \dot{\kappa}(t)$, $\dot{\tau}(t) \in \mathcal{L}_{\infty}$, then Eqs. 14 and 16 can be used to conclude that $F(t), \dot{F}^{T}(t), \ddot{F}^{T}(t) \in \mathcal{L}_{\infty}$.

Remark 1 The partial derivative of $F(t)$ with respect to the arc length of the desired contour is commonly referred to as the Serret-Frenet [19-21] formulas. From the fundamental theorem of space curves [22], the desired contour can be uniquely specified (except in position and orientation) by the single valued continuous functions $\kappa(t)$ and $\tau(t)$.

Remark 2 The following inequalities can be developed from Eqs. 5 to 10 and Property 4:

$$
\begin{aligned}
\|\bar{M}\|_{i \infty} & \leq \zeta_{m} & \left\|\bar{V}_{m}\right\|_{i \infty} \leq \zeta_{v} \\
\|\bar{B}\|_{i \infty} & \leq \zeta_{b} & \|N\|_{i \infty} \leq \zeta_{n}
\end{aligned}
$$

where $\zeta_{m}, \zeta_{v}, \zeta_{b}, \zeta_{n} \in \mathbb{R}$ denote known positive bounding constants.

\section{Control development}

\subsection{Control objective}

The objective in this paper is to develop a CCC for a three-axis CNC machine tool so that a desired contour is followed. To quantify the objective, a contour tracking error, denoted by $e(t) \in \mathbb{R}^{3}$, is defined as follows:

$e \triangleq x_{\mathrm{fd}}-x_{\mathrm{f}}$

where $x_{\mathrm{fd}}(t)$ and $x_{\mathrm{f}}(t)$ are defined in Eqs. 3 and 2 , respectively. To facilitate the subsequent analysis, a filtered tracking error, denoted by $r(t) \in \mathbb{R}^{3}$, is defined as

$r \triangleq \dot{e}+\alpha e$

where $\alpha \in \mathbb{R}$ denotes positive constant control gain. The subsequent control development is based on the assumption that $x_{\mathrm{f}}(t)$ and $\dot{x}_{\mathrm{f}}(t)$ are measurable and that $x_{\mathrm{fd}}(t), \dot{x}_{\mathrm{fd}}(t), \ddot{x}_{\mathrm{fd}}(t), \dddot{x}_{\mathrm{fd}}(t)$ are known and are bounded (and hence $e(t), \dot{e}(t), r(t)$ can be computed).

\subsection{Closed-loop error system}

The open-loop error system for $r(t)$ can be obtained by taking the time derivative of Eq. 20 and premultiplying the resulting expression by $\bar{M}(t)$ as follows:

$\bar{M} \dot{r}=\bar{M}\left(\ddot{x}_{\mathrm{fd}}-\ddot{x}_{\mathrm{f}}\right)+\alpha \bar{M} \dot{e}$.
After utilizing Eqs. 4, 19, and 20, the following expression can be obtained:

$\bar{M} \dot{r}=-\frac{1}{2} \bar{V}_{m} r+Y_{\mathrm{d}} \theta+\chi-\bar{T}$

where the product of the linear regression matrix $Y_{\mathrm{d}}\left(x_{\mathrm{fd}}(t), \dot{x}_{\mathrm{fd}}(t), \ddot{x}_{\mathrm{fd}}(t)\right) \in \mathbb{R}^{3 \times 6}$ and the vector of unknown constants $\theta$ introduced in Eq. 12 is given by the following expression:

$Y_{\mathrm{d}} \theta=\bar{M} \ddot{x}_{\mathrm{fd}}+\bar{V}_{m} \dot{x}_{\mathrm{fd}}+\bar{B} \dot{x}_{\mathrm{fd}}+N x_{\mathrm{fd}}$,

and the auxiliary expression $\chi(e, r) \in \mathbb{R}^{3}$ is defined as

$\chi=\bar{V}_{m} \alpha e+(\alpha \bar{M}-\bar{B})(r-\alpha e)-N e-\frac{1}{2} \bar{V}_{m} r$.

By utilizing Eqs. 17, 18, and 23, the following upper bound can be developed for $\chi(e, r)$ :

$\|\chi\| \leq \zeta_{1}\|z\| \quad$ where $\quad z(t) \triangleq\left[e^{T} r^{T}\right]^{T}$

where $\zeta_{1} \in \mathbb{R}$ is a known, positive bounding constant. Based on Eq. 21 and subsequent stability analysis, $\bar{T}(t)$ is designed as follows:

$\bar{T}=\left(k_{1}+k_{n} \zeta_{1}^{2}\right) r+Y_{\mathrm{d}} \hat{\theta}+e$.

In Eq. 25, $k_{1}, k_{n} \in \mathbb{R}$ denote positive constant control gains, and $\hat{\theta}(t) \in \mathbb{R}^{6}$ denotes an adaptive estimate that is generated from the following differential equation:

$\hat{\theta}=\Gamma Y_{\mathrm{d}}^{T} r \quad \hat{\theta}(0)=\bar{\theta}$

where $\Gamma \in \mathbb{R}^{6 \times 6}$ is a constant diagonal matrix of adaptation gains and $\bar{\theta} \in \mathbb{R}^{6}$ denotes an initial best-guess estimate of the unknown parameters in $\theta$. The closed-loop error system for $r(t)$ can be obtained after substituting Eq. 25 into Eq. 21 as follows:

$\bar{M} \dot{r}=-\frac{1}{2} \bar{V}_{m} r+Y_{\mathrm{d}} \tilde{\theta}+\chi-\left(k_{1}+k_{n} \zeta_{1}^{2}\right) r-e$

where $\tilde{\theta}(t) \in \mathbb{R}^{6}$ denotes the adaptive estimate mismatch defined as follows:

$\tilde{\theta}=\theta-\hat{\theta}$.

\subsection{Stability analysis}

Theorem 1 Provided the control gain $k_{n}$ is selected according to the following sufficient condition:

$k_{n}>\frac{1}{4 \lambda_{1}}$

where $\lambda_{1}=\min \left(k_{1}, \alpha\right)$, the control law given in Eq. 25 ensures global asymptotic tracking control in the sense that

$\lim _{t \rightarrow \infty}\|e(t)\|=0$. 
Proof Let $V(t) \in \mathbb{R}$ denote the following nonnegative Lyapunov function:

$V=\frac{1}{2} r^{T} \bar{M} r+\frac{1}{2} e^{T} e+\frac{1}{2} \widetilde{\theta} \Gamma^{-1} \widetilde{\theta}$.

The time derivative of Eq. 31 can be written as follows:

$\dot{V}=-\alpha e^{T} e-\left(k_{1}+k_{n} \zeta_{1}^{2}\right) r^{T} r+r^{T} \chi$

where Eqs. 11, 20, 26, and 27 were utilized. Based on Eq. 24, the following inequality can be developed for Eq. 32

$\dot{V} \leq-\lambda_{1}\|z\|^{2}+\left[\zeta_{1}\|r\|\|z\|-k_{n} \zeta_{1}^{2}\|r\|^{2}\right]$

where $\lambda_{1}$ was introduced in Eq. 29. The following expression can be obtained after completing the squares on the bracketed terms in Eq. 33

$\dot{V} \leq-\lambda_{1}\|z\|^{2}+\frac{\|z\|^{2}}{4 k_{n}}$.

Provided $k_{n}$ is selected according to the sufficient condition given in Eq. 29, the following inequality can be developed

$\dot{V} \leq-\beta\|z\|^{2}$

for some positive constant $\beta \in \mathbb{R}$. From Eqs. 31 and 35 , we can conclude that $V(t) \in \mathcal{L}_{\infty}$; hence, $e(t), r(t)$, $\tilde{\theta}(t) \in \mathcal{L}_{\infty}$. The expressions in Eqs. 31 and 35 can also be used to conclude that $e(t), r(t) \in \mathcal{L}_{2}$. Given that $e(t)$, $r(t) \in \mathcal{L}_{\infty}$, linear analysis methods can be used to prove that $\dot{e}(t) \in \mathcal{L}_{\infty}$ from Eq. 20. Since $\tilde{\theta}(t) \in \mathcal{L}_{\infty}$, Eq. 28 can be used to prove that $\hat{\theta}(t) \in \mathcal{L}_{\infty}$. The assumption that $x_{\mathrm{fd}}(t), \dot{x}_{\mathrm{fd}}(t), \ddot{x}_{\mathrm{fd}}(t) \in \mathcal{L}_{\infty}$ can be used along with Eqs. 17 and 18 to conclude that $Y_{\mathrm{d}}\left(x_{\mathrm{fd}}(t), \dot{x}_{\mathrm{fd}}(t), \ddot{x}_{\mathrm{fd}}(t)\right) \in \mathcal{L}_{\infty}$. Based on these facts, Eqs. 24-27 can be used to prove that $\chi(e, r), \bar{T}(t), \hat{\theta}(t), \dot{r}(t) \in \mathcal{L}_{\infty}$. Since $e(t), \dot{e}(t), r(t)$, $\dot{r}(t) \in \mathcal{L}_{\infty}$ (i.e., a sufficient condition for $e(t)$ and $r(t)$ to be uniformly continuous) and $e(t), r(t) \in \mathcal{L}_{2}$, Barbalat's Lemma can be used to prove the result in Eq. 30. Since $F(t)$ is a unique transformation, Eqs. 2 and 3 can be used to prove that if the result in Eq. 30 is obtained, then

$x \rightarrow x_{\mathrm{d}} \quad$ as $\quad t \rightarrow \infty$.

\section{Experimental results}

The proposed controller was applied to a feed drive system on a HSM2 developed at the Machine Tool

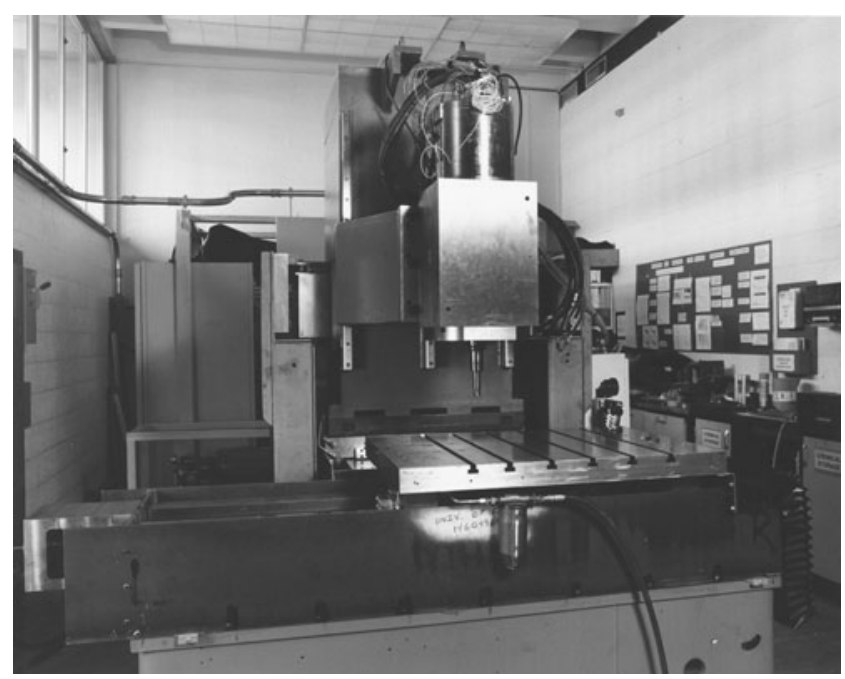

Fig. 2 High speed milling machine

Research Center at the University of Florida (see Fig. 2). For simplicity in result analysis, the contour error produced by the $x$ - and $y$-axes is considered. However, $z$-axis could be easily applied to the proposed controller since the developed control law is based on the $n$-dimensional matrix format. Figure 3 shows the overall configuration of HSM2. The $x$-axis, which supports the workpiece, retains a rotary servo motor, hydrostatic leadscrew and nut, and the table.

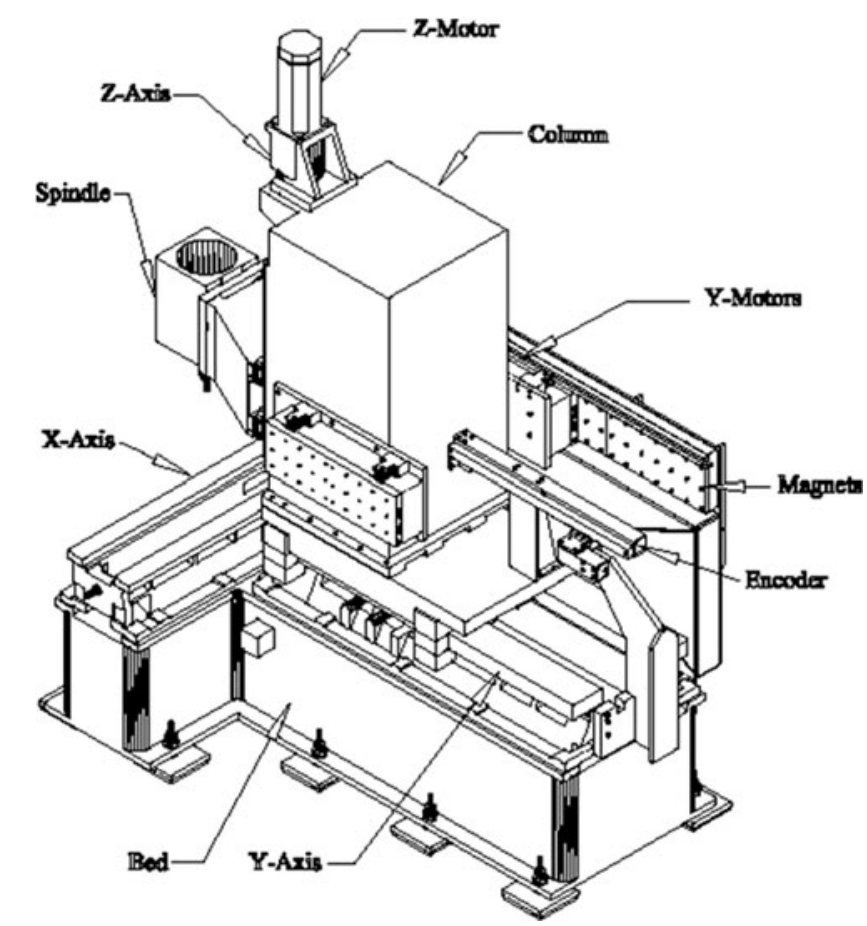

Fig. 3 Configuration of high speed milling machine 
The $y$-axis, which supports the column that contains the $z$-axis and spindle, is driven by two linear motors. As guideways for the $x$ - and $y$-axes, the hydrostatic guideways are used to reduce friction and wear. The Turbo PMAC2 controller from Delta-Tau Inc. is used as a controller which performs the phase commutation for the servomotors and closes the current loop digitally providing the pulse width modulation format for the motor power with $1 \mathrm{~ms}$ servo update time. To implement the proposed controller on this controller, the user-written servo algorithm provided by the Turbo PMAC2 controller is used. Position feedback for the $x$-axis is provided by a rotary encoder which translates to a linear resolution of $2 \mu \mathrm{m}$. A single linear encoder with $1 \mu \mathrm{m}$ resolution attached to the end of the column is used for the $y$-axis position feedback.

The dynamics for the $x$ - and $y$-axes of HSM2 are given as follows:

$\left[\begin{array}{cc}M_{x 1} & 0 \\ 0 & M_{x 2}\end{array}\right]\left[\begin{array}{l}\ddot{x}_{1} \\ \ddot{x}_{2}\end{array}\right]+\left[\begin{array}{cc}B_{x 1} & 0 \\ 0 & B_{x 2}\end{array}\right]\left[\begin{array}{l}\dot{x}_{1} \\ \dot{x}_{2}\end{array}\right]=T$

where $M_{x 1}=0.05916 \mathrm{~kg} \mathrm{~m}^{2}$ denotes the equivalent moment of inertia of the $x$-axis, $M_{x 2}=1,144 \mathrm{~kg}$ denotes the moving mass of the $y$-axis, $B_{x 1}=0.01725 \mathrm{~N} \mathrm{~m} / \mathrm{rad} / \mathrm{s}$ denotes the friction of the $x$-axis, and $B_{x 2}=967 \mathrm{~N} / \mathrm{m} / \mathrm{s}$ denotes the friction of the $y$-axis. These system parameters are obtained through the system identification procedure and verified by comparison between simulation and experiment [23]. For the initial best-guess estimate of unknown parameters in Eq. 26, these values are used. Three circular trajectories on the $x-y$-axes of HSM2 are used to examine the performance of the proposed controller (see Table 1). For circular motions, the contour error $\varepsilon$ is calculated as $\varepsilon=$ $\sqrt{\left(p_{x}-x_{0}\right)^{2}+\left(p_{y}-y_{0}\right)^{2}-\rho}$ where $\rho \in \mathbb{R}$ is the circle radius, $\left(x_{0}, y_{0}\right)$ is the corresponding center of the circle, and $\left(p_{x}(t), p_{y}(t)\right)$ is the actual tool position.

Since the HSM2 $x-y$ feed drive system is a 2-D contouring system, the transformation matrix $F(t)$ is represented by

$F(t)=\left[\begin{array}{cc}\cos \theta(t) & -\sin \theta(t) \\ \sin \theta(t) & \cos \theta(t)\end{array}\right]$

Table 1 Specification of trajectories

\begin{tabular}{llll}
\hline Case & $\begin{array}{l}\text { Radius } \\
(\mathrm{m})\end{array}$ & $\begin{array}{l}\text { Feedrate } \\
(\mathrm{m} / \mathrm{s})\end{array}$ & $\begin{array}{l}\text { Acceleration } \\
\left(\mathrm{m} / \mathrm{s}^{2}\right)\end{array}$ \\
\hline I & 0.1 & 0.2 & 0.4 \\
II & 0.1 & 0.4 & 1.6 \\
III & 0.06 & 0.4 & 2.67 \\
\hline
\end{tabular}

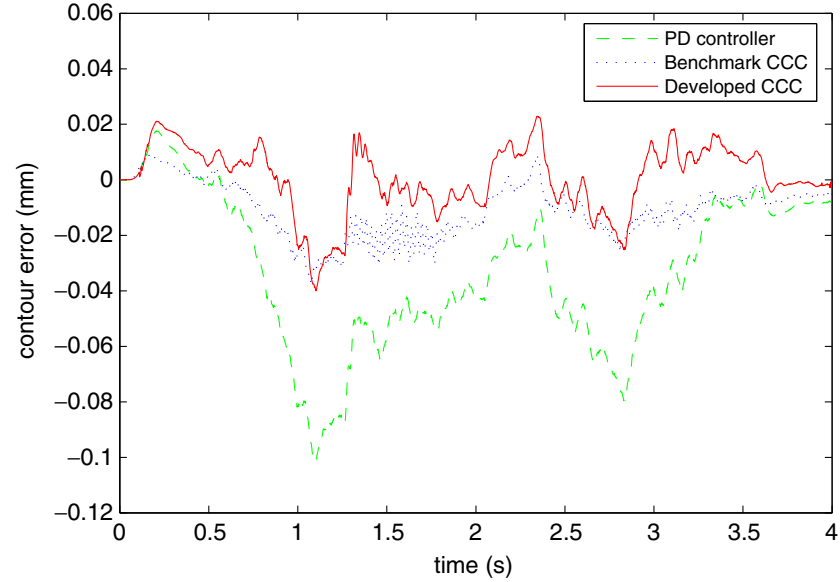

Fig. 4 Contour error at $0.2 \mathrm{~m} / \mathrm{s}$ with $0.1 \mathrm{~m}$ radius (case I)

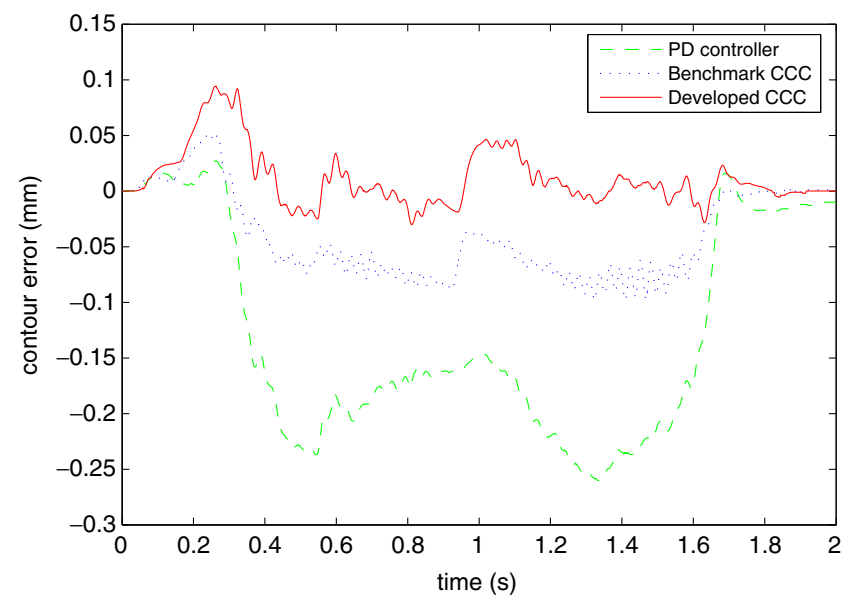

Fig. 5 Contour error at $0.4 \mathrm{~m} / \mathrm{s}$ with $0.1 \mathrm{~m}$ radius (case II)

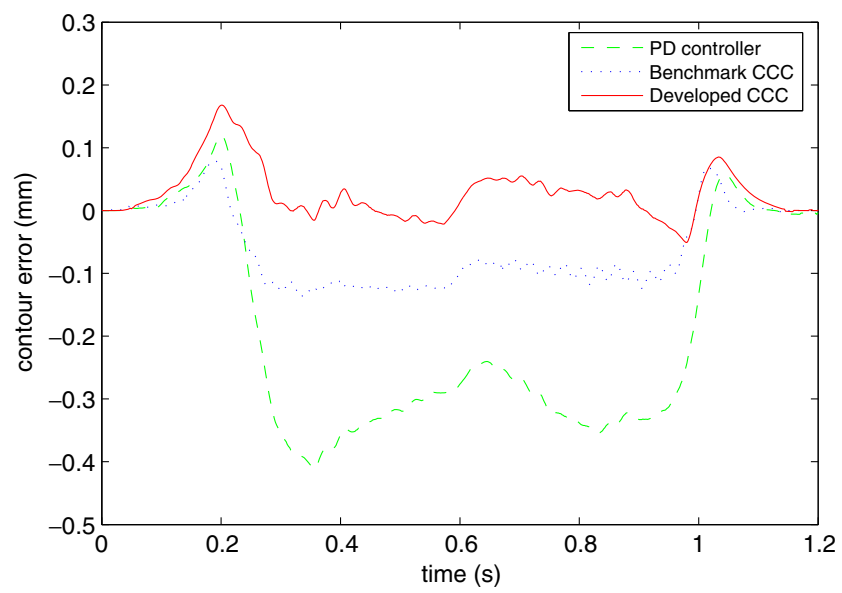

Fig. 6 Contour error at $0.4 \mathrm{~m} / \mathrm{s}$ with $0.06 \mathrm{~m}$ radius (case III) 
Fig. 7 Control effort at $0.2 \mathrm{~m} / \mathrm{s}$ with $0.1 \mathrm{~m}$ radius (case I)
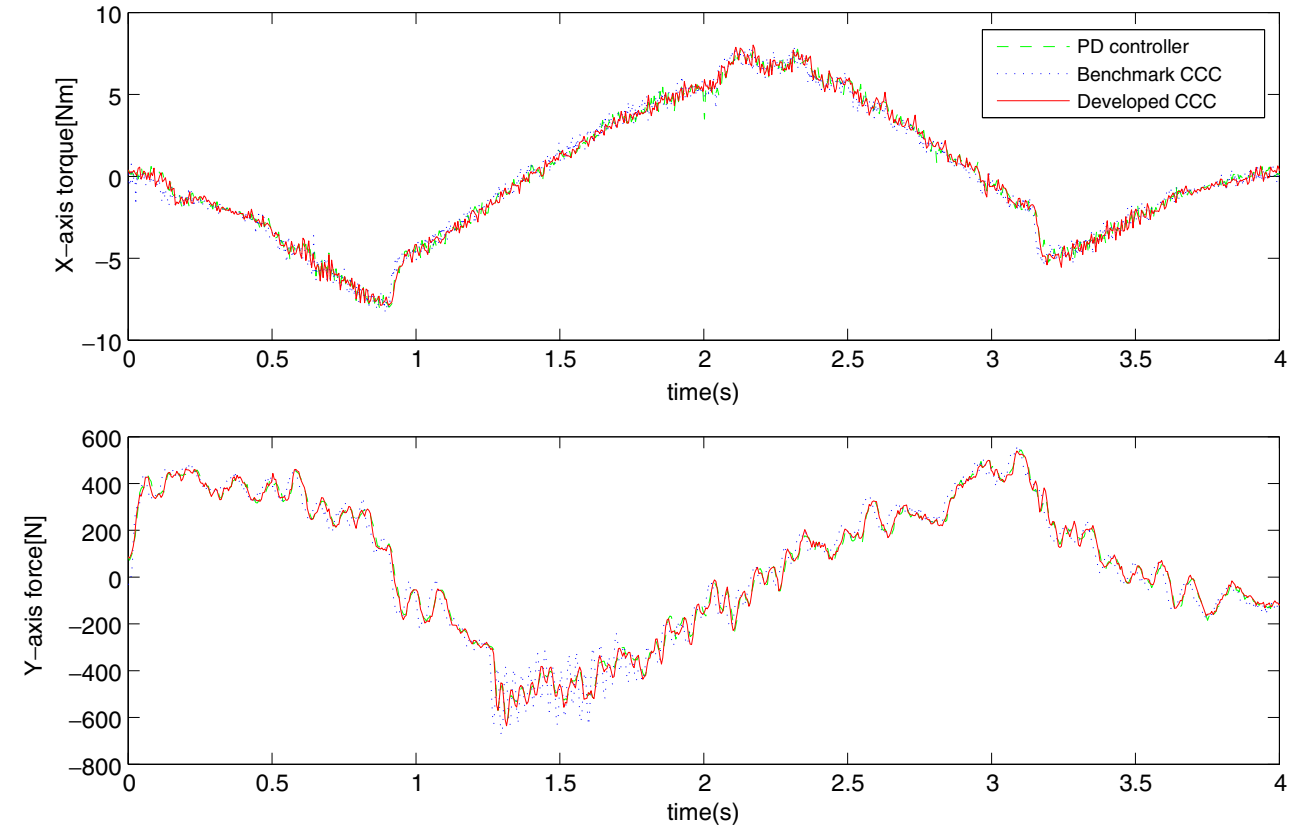

where $\theta(t)$ is defined as the incline angle between the desired velocity vector and the $x$-axis. For a 2-D circular contour,

$R=\left[\begin{array}{cc}0 & \frac{1}{\rho} \\ -\frac{1}{\rho} & 0\end{array}\right]$

where $\rho$ is the circle radius.
The control force $T(t)$ in Eqs. 1, 9, and 25 can be expressed as

$$
\begin{aligned}
T= & k v\left(F R F^{T}\right)\left(x_{\mathrm{d}}-x\right)+(\alpha k+1)\left(x_{\mathrm{d}}-x\right) \\
& +k\left(\dot{x_{\mathrm{d}}}-\dot{x}\right)+F\left(Y_{\mathrm{d}} \hat{\theta}\right)
\end{aligned}
$$

where $k=\left(k_{1}+k_{n} \zeta_{1}^{2}\right)$, and Eqs. 2, 3, 9, 14, 19, and 20 have been utilized. The control force in Eq. 38 can be described as a cross-coupling component (i.e., the first set of terms), a proportional component (i.e., the second set of terms), a derivative component (i.e., the third set of terms), and the adaptive
Fig. 8 Control effort at $0.4 \mathrm{~m} / \mathrm{s}$ with $0.1 \mathrm{~m}$ radius (case II)
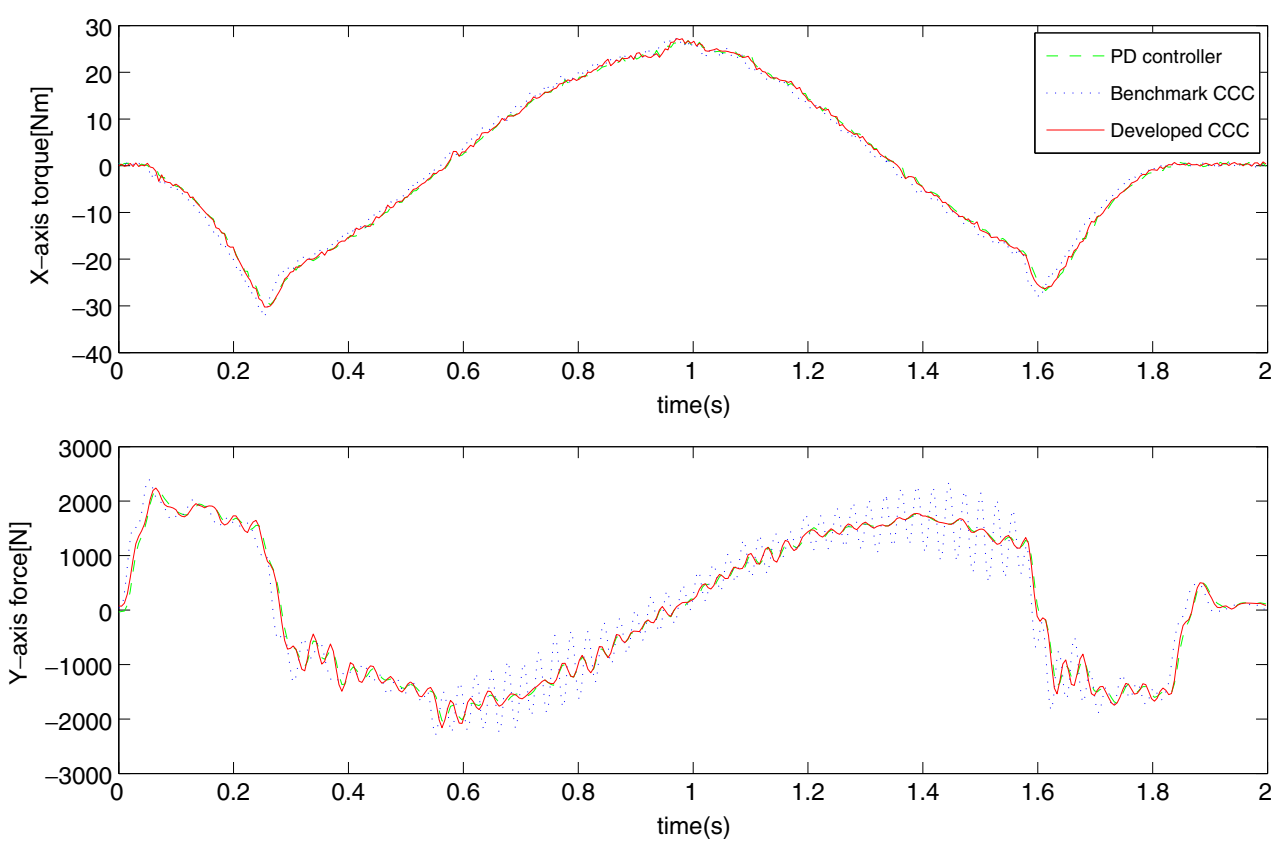
Fig. 9 Control effort at $0.4 \mathrm{~m} / \mathrm{s}$ with $0.06 \mathrm{~m}$ radius (case III)
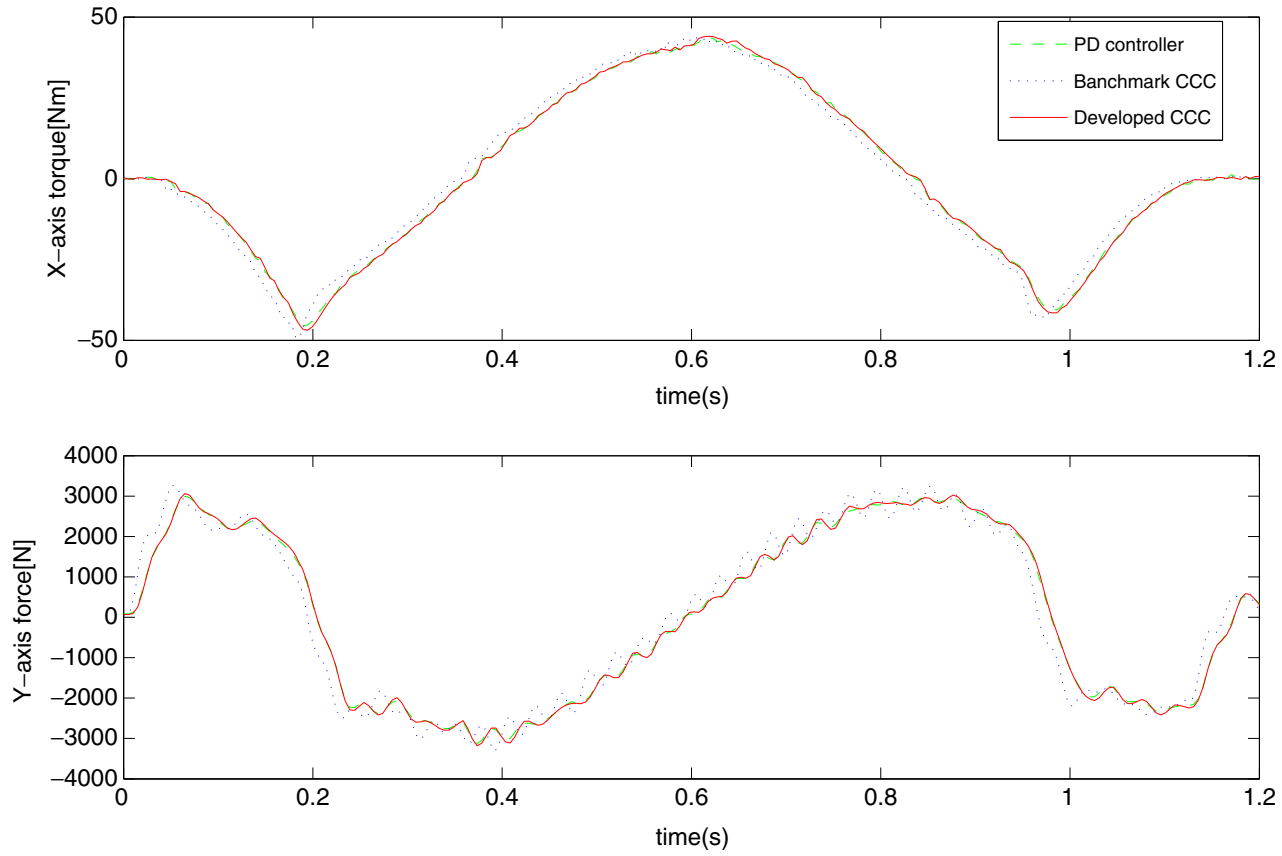

feedforward component (i.e., the fourth set of terms). For the 2-D circular contour, Eqs. 36 and 37 can be used to express the cross-coupling component of Eq. 38 as

$$
\begin{aligned}
& k v\left(F R F^{T}\right)\left(x_{\mathrm{d}}-x\right) \\
& =\left[\begin{array}{cc}
\left(\frac{k v}{\rho}\right)_{1} & 0 \\
0 & \left(\frac{k v}{\rho}\right)_{2}
\end{array}\right]\left[\begin{array}{c}
x_{\mathrm{d} 2}-x_{2} \\
-\left(x_{\mathrm{d} 1}-x_{1}\right)
\end{array}\right] .
\end{aligned}
$$

Contour errors obtained from implementing a PD controller and the benchmark CCC developed in [4] are also examined for comparison. The axial gains for PD controller are determined as

$P_{x}=360,000, D_{x}=9,000,000$,

$P_{y}=58,000, D_{y}=1,450,000$.

To select the PD gains, firstly for each axis, the best gain which results in the lowest following error with-

Table 2 comparison of contouring performance

\begin{tabular}{llcc}
\hline Case & Controller & $\|\varepsilon\|_{\text {MAX }}(\mu \mathrm{m})$ & $\varepsilon_{\text {RMS }}(\mu \mathrm{m})$ \\
\hline I & PD & 119 & 43 \\
& Benchmark CCC & 46 & 15 \\
& Developed CCC & 63 & 12 \\
II & PD & 288 & 160 \\
& Benchmark CCC & 149 & 56 \\
& Developed CCC & 125 & 28 \\
III & PD & 530 & 250 \\
& Benchmark CCC & 215 & 86 \\
& Developed CCC & 219 & 49 \\
\hline
\end{tabular}

out overshoot is chosen by gradually increasing the gain. Then, by balancing the gains between the $X$-and $Y$-axes, the final PD gain which results in the minimal contour error is determined. For the benchmark CCC, the proportional control law is used and the best gain is determined as 3,584. Since the second and third terms in Eq. 38 represent the PD controller, the existing PD controller is used for those. The gains that resulted in the best performance in circular contours are determined as follows:

$\left(\frac{k v}{\rho}\right)_{1}=422,\left(\frac{k v}{\rho}\right)_{2}=1359$,

$\Gamma=\left[\begin{array}{cccc}5 \times 10^{-2} & 0 & 0 & 0 \\ 0 & 5 \times 10^{-2} & 0 & 0 \\ 0 & 0 & 1 & 0 \\ 0 & 0 & 0 & 1\end{array}\right]$

Table 3 comparison of control effort

\begin{tabular}{lllc}
\hline Case & Controller & $\begin{array}{l}X \text {-axis torque } \\
\left(T_{\text {RMS }}(\mathrm{N} \mathrm{m})\right)\end{array}$ & $\begin{array}{c}Y \text {-axis force } \\
\left(F_{\text {RMS }}(\mathrm{N})\right)\end{array}$ \\
\hline I & PD & 3.85 & 287 \\
& Benchmark CCC & 3.83 & 293 \\
& Developed CCC & 3.86 & 288 \\
II & PD & 15.2 & 1,270 \\
& Benchmark CCC & 15.3 & 1,310 \\
& Developed CCC & 15.3 & 1,280 \\
III & PD & 24.0 & 1,980 \\
& Benchmark CCC & 24.2 & 2,010 \\
& Developed CCC & 24.2 & 1,990 \\
\hline
\end{tabular}


Since the maximum and the average contouring errors are the main contributors to the quality of the final part, the maximum absolute contouring error $\|\varepsilon(t)\|_{\text {MAX }}$ and the root mean square (RMS) contouring error $\varepsilon_{\mathrm{RMS}}(t)$ are used to compare the performance of each control algorithm. Figure 4 shows the contouring error for case I. While the RMS contouring error for the PD controller is $43 \mu \mathrm{m}$, the RMS contouring error for the benchmark CCC and proposed CCC are 15 and $12 \mu \mathrm{m}$, respectively. Figures 5 and 6 indicate an improvement in contouring performance by the proposed control algorithm as the feedrate and acceleration increase when compared to [4]. This improvement is attributed to the fact that the proposed CCC accounts for uncertain nonlinear terms through the adaptive feedforward component. The control efforts in each case are shown in Figs. 7, 8, and 9, respectively, which accounts for the economic feasibility of the developed CCC. Table 2 summarizes the circular contouring performance of each controller, and Table 3 compares the respective control effort.

\section{Conclusion}

In this paper, Lyapunov-based controllers are developed for a three-axis CNC machine tool feed drive system. To mitigate contour errors, the control development was based on an error system that was transformed into tangential, normal, and binormal components to the desired contour. The contribution of the results over previous CCCs are that uncertainty in the machine tool dynamics is considered. Specifically, an adaptive estimate was developed to compensate for uncertain inertia and friction parameters. The proposed control law was implemented on the feed drive system of HSM2, and experimental results showed improvements in contouring performance over the existing PD controller and the benchmark CCC. Ongoing efforts are focused on semi-global asymptotic contour following in the presence of uncertain friction and cutting force disturbances on HSM2.

\section{References}

1. Tomizuka M (1987) Zero phase error tracking algorithm for digital control. J Dyn Syst Meas Control 109:65-68

2. Weck M, Ye G (1990) Sharp corner tracking using the IKF control strategy. Ann CIRP 39(1):437-441
3. Koren Y (1980) Cross-coupled biaxial computer control for manufacturing systems. ASME Trans J Dyn Syst Meas Control 102(4):265-272

4. Koren Y, Lo CC (1991) Variable-gain cross-coupling controller for contouring. Ann CIRP 104:371-374

5. Yeh S-S, Hus P-L (1999) Analysis and design of the integrated controller for precise motion systems. IEEE Trans Control Syst Technol 7(6):706-717

6. Yeh S-S, Hus P-L (2003) Analysis and design of integrated control for multi-axis motion systems. IEEE Trans Control Syst Technol 11(3):375-382

7. Yeh S-S, Hus P-L (2002) Estimation of the contouring error vector for the cross-coupled control design. IEEE Trans Mechatron 7(1):44-51

8. Kulkarni PK, Srinivasan K (1989) Optimal contouring control of multi-axial drive servomechanisms. ASME Trans J Eng Ind 111:140-148

9. Srinivasan K, Kulkarni PK (1990) Cross-coupled control of biaxial feed drive servomechanisms. ASME Trans J Dyn Syst Meas Control 112(2):225-232

10. Chen S-L, Liu H-L, Ting SC (2002) Contouring control of biaxial systems based on polar coordinates. IEEE/ASME Trans Mechatron 7(3):329-345

11. Yeh Z-M, Tarng YS, Lin YS (1997) Cross-coupled fuzzy logic control for multiaxis machine tools. Mechatronics 7(8):663681

12. Tarng YS, Chuang HY, Hus WT (1999) Intelligent crosscoupled fuzzy federate controller design for CNC machine tools based on genetic algorithms. Int J Mach Tool Manuf 39:1673-1692

13. Chuang HY, Liu CH (1991) Cross-coupled adaptive feedrate control for multiaxis machine tools. ASME Trans J Dyn Syst Meas Control 113:451-457

14. Tomizuka M, Hu J, Chiu T-C, Kamano T (1992) Synchronization of two motion control axes under adaptive feedforward control. ASME Trans J Dyn Syst Meas Control 114:196203

15. Chiu GT-C, Tomizuka M (2001) Contouring control of machine tool feed drive systems: a task coordinate frame approach. IEEE Trans Control Syst Technol 9(1):130-139

16. Chiu GT-C, Yao B (1997) Adaptive robust control tracking of machine tool feed drive system-a task coordinate frame approach. In: Proc. of the American control conf., Albuquerque, New Mexico, pp 2731-2735

17. Xu L, Yao B (2000) Coordinated adaptive robust contour tracking of linear-motor-driven tables in task space. In: Proc. of the 39th IEEE conf. on decision and control, Sydney, Australia, pp 2430-2435

18. Lewis F, Abdallah C, Dawson D (1993) Control of robot manipulators. MacMillan, New York

19. Frenet F (1847) Sur les courbes à double courbure, Thèse, Toulouse. Abstract in J de Math, vol 17 (1852)

20. Kreyszig E (1991) Formulae of Frenet. In: Differential geometry. Dover, New York, pp 40-43

21. Serret JA (1851) Sur quelques formules relatives à la théorie des courbes à double courbure. J de Math 16

22. Gray A (1997) The fundamental theorem of space curves, modern differential geometry of curves and surfaces with mathematica, 2nd edn. CRC, Boca Raton, pp 219-222

23. Lee J (2005) Design of controllers for improving contour accuracy in a high-speed milling machine. Ph.D. dissertation, Univ. Florida 